\title{
THE THIRRING MODEL FROM THE XXZ HEISENBERG CHAIN SCHWINGER TERMS
}

\author{
H.J. DE VEGA and T.J.M. SIMÕES 1 \\ Laboratoire de Physique Théorique et Hautes Energies ${ }^{2}$, \\ Universté Paris VI, Tour 16, Ier. étage, 4 Place Jussieu, 75252 Paris Cedex 05, France
}

Received 25 October 1988

\begin{abstract}
The propertes of the Thirring model solution obtained from the XXZ Heisenberg chain are computed exactly using the Bethe Ansatz. The fermion fields, vector current, axial anomaly and equal-time current commutators are constructed explicitly on the lattice and then its continuous limit is obtained. An explicit coupling constant dependence is found for the Schwinger term. This result, together with the current correlation behavior and the conformal weights of the fields here, shows that this Thirring model solution is new. That is, it is a spin $\frac{1}{2}$ solution not contained in previous families of continuous solutions.
\end{abstract}

In spite of its wide success to solve exactly a large set of interacting QFT ${ }^{\# 1}$, anomalies are usually outside the scope of the Bethe Ansatz (BA) and its generalizations. In the present note we find the Schwinger term and investigate related properties of the massless Thirring model using the BA method on the lattice. Our starting point is the XXZ Heisenberg model which yields the Thirring model in the continuum limit through the Jordan-Wigner transformation $[4]$

The XXZ Heisenberg hamiltonian for a chain of $N$ spins is given by

$H_{\mathrm{XXz}}=-\frac{1}{4 a} \sum_{n=1}^{N}\left(\sigma_{n}^{x} \sigma_{n+1}^{x}+\sigma_{n}^{y} \sigma_{n+1}^{y}+\Delta \sigma_{n}^{z} \partial_{n+1}^{z}\right)$.

Here $\sigma_{n}^{a}(a=x, y, z ; 1 \leqslant n \leqslant N)$ are Pauli matrices acting at the $n$th site, $\gamma$ is the anisotropy parameter and $a$ the lattice spacing. The factor $a^{-1}$ has been introduced to give mass dimensions to the hamiltonian.

The Jordan-Wigner transformation expresses lattice fermion operators in terms of spin $\frac{1}{2}$ operators

$\phi_{n}=\mathrm{i}^{n} \sigma_{n}^{-} \prod_{l=1}^{N-1} \sigma_{\bar{l}}, \quad \phi_{n}^{+}=\mathrm{i}^{-n} \sigma_{n}^{+} \prod_{l=1}^{N-1} \sigma_{\bar{l}}$.

1 Permanent address: Instituto de Fisica, Universidad Federal de Rio Grande do Sul 90049, Porto Alegre, RS, Brasil.

2 Laboratoire associé au CNRS, UA 280.

\#1 For reviews, see refs. [1-3].
They fulfill canonical anticommutation relations.

$$
\left\{\phi_{n}, \phi_{m}^{\dagger}\right\}=\delta_{n m}, \quad\left\{\phi_{n}, \phi_{m}\right\}=0 \text {. }
$$

The XXZ hamiltonian (1) is written in terms of these lattice fermions as [4]

$$
\begin{gathered}
H_{\mathrm{XXZ}}=\frac{1}{a} \sum_{n=1}^{N}\left[-\frac{1}{2} \mathrm{i}\left(\phi_{n}^{\dagger} \phi_{n+1}-\phi_{n+1}^{\dagger} \phi_{n}\right)\right. \\
\left.-\Delta\left(\phi_{n}^{\dagger} \phi_{n}-\frac{1}{2}\right)\left(\phi_{n+1}^{\dagger} \phi_{n+1}-\frac{1}{2}\right)\right] .
\end{gathered}
$$

The resulting lattice equations of motion are

$$
\begin{gathered}
\left.\partial_{0} \phi_{n}=\mathrm{i}\left[H_{\mathrm{XXZ}}, \phi_{n}\right]=-(1 / 2 a) \phi_{n+1}-\phi_{n-1}\right) \\
-(\mathrm{i} J / a) \phi_{n}\left[1-\phi_{n-1}^{\dagger} \phi_{n-1}-\phi_{n+1}^{\dagger} \phi_{n+1}\right] .
\end{gathered}
$$

It is convenient to consider the $\phi_{n}$ as KogutSusskind fermions. That is, we set

$$
\begin{aligned}
& \Psi_{1}(m)=(2 a)^{-1 / 2} \phi_{2 m}, \\
& \Psi_{2}(m)=(2 a)^{-1 / 2} \phi_{2 m+1}
\end{aligned}
$$

for the two components of the fermion.

After a trivial phase redefinition $\Psi \rightarrow$ $\exp \left(-2 \mathrm{i} \Delta x_{0}\right) \Psi$, the continuum limit for the hamiltonian follows straightforward:

$$
\begin{aligned}
& \lim _{a \rightarrow 0} H_{\mathrm{XXZ}}=\int \mathrm{d} x\left[-\mathrm{i}\left(\Psi_{1}^{\dagger} \partial_{x} \Psi_{2}+\Psi_{2}^{\dagger} \partial_{x} \Psi_{1}\right)\right. \\
& \left.-2 g \Psi_{1}^{\dagger} \Psi_{2}^{\dagger} \Psi_{1} \Psi_{2}\right] \equiv H_{\mathrm{TM}}
\end{aligned}
$$

and for the equations of motion 
$\mathrm{i} \not \partial \Psi=g \gamma^{\mu} J_{\mu} \Psi$,

where $x=n a, g \equiv-24$ is the Thirring coupling constant and the Dirac matrix representation used here reads

$\gamma^{0}=\sigma^{3}, \quad \gamma^{1}=\mathrm{i} \sigma^{2}, \quad \gamma^{5}=\gamma^{0} \gamma^{1}=\sigma^{1}$.

That is, the vector current components are

$J^{0}=\Psi_{1}^{\dagger} \Psi_{1}+\Psi_{2}^{\ddagger} \Psi_{2}, \quad J^{1}=\Psi_{1}^{\dagger} \Psi_{2}+\Psi_{2}^{\dagger} \Psi_{1}$

in the continuum limit.

Let us find the lattice version of this vector current. We define

$$
\begin{gathered}
J_{0}(n)=(1 / 2 a)\left(\phi_{n}^{\dagger} \phi_{n}+\phi_{n+1}^{\dagger} \phi_{n+1}\right) \\
=(1 / 2 a)\left[1+\frac{1}{2}\left(\sigma_{n}^{z}+\sigma_{n+1}^{z}\right)\right] .
\end{gathered}
$$

Then, we get from eq. (4)

$$
\partial_{0} J_{0}(n)=+(1 / 2 a)\left[J_{1}(n-1)-J_{1}(n+1)\right]
$$

or

$\partial_{0} J_{0}(n)+\Delta_{x} J_{1}(n)=0$,

where

$J_{1}(n) \equiv(1 / 2 a)\left(\phi_{n}^{\dagger} \phi_{n+1}+\phi_{n+1}^{\dagger} \phi_{n}\right)$

and

$\Delta_{x} A(n) \equiv(1 / 2 a)[A(n+1)-A(n-1)]$.

Therefore we have an exact conserved vector current on the lattice $\left(J_{0}(n), J_{1}(n)\right)$.

Notice that the associated conserved charge is just the $z$-component of the total spin.

$Q=\sum_{n} J_{0}(n)=\frac{1}{2 a} \sum_{n} \sigma_{n}^{z}+\frac{N}{2 a}$.

When $a \rightarrow 0$ the current conservation equation reads

$\partial_{0} J_{0}(x)+\partial_{1} J_{1}(x)=0$

as it should be. In the continuum limit the present lattice construction becomes manifestly relativistic invariant as one sees from eqs. (7), (8) and (16).

The continuum Thirring model usually possesses in addition a conserved axial current $J_{\mu}^{5}(x)=$ $\varepsilon_{\mu \nu} J^{\nu}(x)[5]$.

Its lattice version is not conserved here for $a>0$. We find from eq. (4)

$$
\begin{aligned}
& \partial_{0} J_{1}(n)+\Delta_{x} J_{0}(n) \\
& \quad=-\left(\Delta / 8 a^{2}\right)\left(\sigma_{n}^{x} \sigma_{n+1}^{x}+\sigma_{n}^{y} \sigma_{n+1}^{y}\right)\left(\sigma_{n-1}^{z}-\sigma_{n+2}^{z}\right),
\end{aligned}
$$

a clearly non-zero result.

The current-field commutators on the lattice follow from eqs. (3), (11) and (13):

$$
\begin{aligned}
& {\left[J_{0}(n), \phi_{m}\right]=-(1 / 2 a)\left(\delta_{n m}+\delta_{n, m-1}\right) \phi_{m},} \\
& {\left[J_{1}(n), \phi_{m}\right]=-(1 / 2 a)\left(\delta_{n m} \phi_{m+1}+\delta_{n, m-1} \phi_{m-1}\right) .}
\end{aligned}
$$

From eqs. (6), (18) we find in the continuum limit the canonical result

$$
\begin{aligned}
& {\left[J_{0}(x), \Psi(y)\right]=-\delta(x-y) \Psi(y),} \\
& {\left[J_{1}(x), \Psi(y)\right]=-\delta(x-y) \gamma^{5} \Psi(y) .}
\end{aligned}
$$

The integrable $X X Z$ chain possesses an abelian gauge invariance on the lattice [6]. That is the gauge transformed hamiltonian

$$
\begin{aligned}
& H[\alpha]=-\frac{1}{4 a} \sum_{n=1}^{N} \exp \left(-\mathrm{i} \alpha_{n+1} \sigma_{n+1}^{z}\right) \\
& \quad \times\left[\sigma_{n}^{x} \sigma_{n+1}^{x}+\sigma_{n}^{y} \sigma_{n+1}^{v}+\Delta \sigma_{n}^{z} \sigma_{n+1}^{z}\right] \exp \left(\mathrm{i} \alpha_{n+1} \sigma_{n+1}^{z}\right)
\end{aligned}
$$

has the same spectrum as the original one [eq. (1)] for any choice of the gauge parameters

$\alpha \equiv\left(\alpha_{1}, \alpha_{2}, \ldots, \alpha_{N}\right)$.

Moreover, the effect of the gauge transformation is identical to a twist

$\Phi=\sum_{j=1}^{N} \alpha_{j}$

in the boundary conditions [2]. That is

$\sigma_{N+1}^{ \pm}=\exp ( \pm \mathrm{i} \Phi) \sigma_{1}^{ \pm}, \quad \sigma_{N+1}^{\bar{*}}=\sigma_{\mathrm{i}}^{z}$.

Upon the fermionization (2) the gauge transformed XXZ hamiltonian (20) becomes

$$
\begin{aligned}
& H[\alpha]=H[\mathbf{0}] \\
& \quad+\frac{1}{2 a} \sum_{n=1}^{N}\left(a_{n} \phi_{n+1}^{\dagger} \phi_{n}+a_{n}^{*} \phi_{n}^{\dagger} \phi_{n+1}^{\dagger}\right),
\end{aligned}
$$

where

$a_{n}=\sin \left(2 \alpha_{n+1}\right)+2 \mathrm{i} \sin ^{2}\left(\alpha_{n+1}\right)$. 
In the continuum limit this is equivalent to a standard $U(1)$ gauge transformation on the Thirring fields $\Psi(x) \rightarrow \Psi(x) \exp [\mathrm{i} \lambda(x)]$ which adds an extra term to the lagrangian:

$$
\mathscr{L}[\lambda(x)]=\mathscr{L}[0]+\mathrm{i} \bar{\Psi}(\not \lambda) \Psi(x),
$$

where $\lambda^{\prime}(x)=\lim \left(\alpha_{n} / a\right)$ for $x=2 n a$.

Therefore, the lattice gauge transformation [eq. (20) ] on the XXZ chain becomes a usual U (1) gauge transformation on the (continuum) Thirring field $\Psi(x)$. Notice that $\lambda(x)$ is a c number time independent function. So, coupling the Thirring model with a pure gauge electromagnetic field leaves the physics clearly unchanged when $N=\infty$.

Let us now consider the current commutators. At equal times we obtain them on the lattice from eqs. (11), (13) and (3),

$$
\begin{aligned}
& {\left[J^{0}(n), J^{1}(m)\right]=\left(1 / 4 a^{2}\right)\left(\delta_{n, m+1}-\delta_{n, m-1}\right)} \\
& \quad \times\left(\phi_{m+1}^{\dagger} \phi_{m}-\text { h.c. }\right) \\
& \quad=\left(\mathrm{i} / 8 a^{2}\right)\left(\delta_{n, m+1}-\delta_{n, m-1}\right) \\
& \quad \times\left(\sigma_{m}^{\mathrm{r}} \sigma_{m+1}^{\mathrm{x}}+\sigma_{m}^{\mathrm{v}} \sigma_{m+1}^{\mathrm{v}}\right) \\
& {\left[J^{0}(n), J^{0}(m)\right]=0} \\
& {\left[J^{1}(n), J^{1}(m)\right]} \\
& \quad=\left(1 / 4 a^{2}\right)\left(\delta_{n, m+1} \phi_{m+2}^{\dagger} \phi_{m}\right. \\
& \left.\quad-\delta_{n, m-1} \phi_{m+1}^{\dagger} \phi_{n-1}-\text { h.c. }\right)
\end{aligned}
$$

We find that the equal-time commutators on the lattice are operators. The continuum limit is a subtle point.

The ETC (25) in the $a \rightarrow 0$ limit has the form

$$
\mathrm{i} \delta^{\prime}(x-y)\left(\sigma_{m}^{x} \sigma_{m+1}^{x}+\sigma_{m}^{v} \sigma_{m+1}^{v}\right) \text {, }
$$

where $x=2 n a$ and $y=2 m a$. The vacuum expectation value (VEV) of the operator in the RHS will be of order one even for $a \rightarrow 0$ and $N \rightarrow \infty$. Its expectation value on excited states can differ by contributions of order $1 / N$ from the VEV. Therefore, it is enough to compute the VEV and then let $N \rightarrow \infty$ and $a \rightarrow 0$.

The expectation value of the commutators (25)(27) in the ferromagnetic (bare) vacuum

$\Omega=\left(\begin{array}{l}1 \\ 0\end{array}\right) \otimes\left(\begin{array}{l}1 \\ 0\end{array}\right) \otimes \ldots \otimes\left(\begin{array}{l}1 \\ 0\end{array}\right)$

is clearly zero. $\Omega$ is the ground state for $\Delta>1$. When
$\Delta<1$ the ground state is antiferromagnetic and it has been constructed exactly by Bethe Ansatz $\$ 2$.

The eigenvalues $E(A)$ of $H_{\mathrm{xxz}}$ [eq. (1)] are explicitly known for $N=\infty$ (finite $N$ corrections can be found in refs. $[2,7])$.

Let us relate the VEV of eq. (25) with the ground state eigenvalue of $H_{\mathrm{Xxz}}$. Since the ground state is translationally invariant

$$
\begin{aligned}
- & (1 / 2 a)\left\langle 0\left|\sigma_{n}^{x} \sigma_{n+1}^{x}+\sigma_{n}^{y} \sigma_{n+1}^{\nu}+\Delta \sigma_{n}^{z} \sigma_{n+1}^{z}\right| 0\right\rangle \\
= & N^{-1} E(\Delta) .
\end{aligned}
$$

Moreover [8]

$$
\begin{aligned}
\frac{\partial E}{\partial A} & =\left\langle 0\left|\frac{\partial H}{\partial \Delta}\right| 0\right\rangle \\
& =-\frac{1}{2 a}\left\langle 0\left|\sum_{n=1}^{N} \sigma_{n}^{z} \sigma_{n+1}^{z}\right| 0\right\rangle \\
& =-(N / 2 a)\left\langle 0\left|\sigma_{n}^{z} \sigma_{n+1}^{z}\right| 0\right\rangle .
\end{aligned}
$$

Therefore,

$$
\begin{gathered}
Z(\mu) / \pi \equiv\left\langle 0\left|\sigma_{n}^{x} \sigma_{n+1}^{x}+\sigma_{n}^{y} \sigma_{n+1}^{y}\right| 0\right\rangle \\
\quad=-(2 a / N)[1-\Delta(\partial / \partial \Delta)] E(\Delta),
\end{gathered}
$$

where $A=-\cos \mu, 0<\mu<\pi$.

This completes partial results of ref. (9). The ground state energy writes for $N=\infty$ within our normalization (1)

$E(\Delta)=(N / a)\left[\frac{1}{2} \cos \mu-2(\sin \mu / \mu) Y(\mu)\right]$,

where $[10]$

$Y(\mu)=\int_{0}^{\infty} \mathrm{d} y\left(1-\frac{\operatorname{th} y}{\operatorname{th}(\pi y / \mu)}\right)$.

We find from eqs. (32)-(34)

$\frac{Z(\mu)}{\pi}=-2 \frac{\sin 2 \mu-2 \mu}{\mu^{2} \sin \mu} Y(\mu)+\frac{2 \cos \mu}{\mu} \frac{\partial Y}{\partial \mu}$,

and now the conimuum limit of the Schwinger term results from eqs. (25), (28) and (32)

$\left[J^{0}(x), J^{1}(y)\right]=(\mathrm{i} / \pi) Z(\mu) \delta^{\prime}(x-y)$.

The expansion of $Z(\mu)$ around $\mu=\frac{1}{2} \pi$ (where the $\mathrm{XXZ}$ chain becomes a free model) reads

\#2 See e.g. ref. [2]. 
$Z(\mu) \underset{\mu \rightarrow \pi / 2}{=} 1-\left(\frac{17}{18}+\frac{4}{3} \pi^{-2}\right)\left(\mu-\frac{1}{2} \pi\right)^{2}+O\left(\mu-\frac{1}{2} \pi\right)^{3}$.

Let us now consider the space-space commutator (24). It can be recast using eq. (2) as

$$
\begin{aligned}
& {\left[J^{1}(n), J^{1}(m)\right]} \\
& \quad=\left(\mathrm{i} / 8 a^{2}\right)\left(\delta_{n, m+1} \delta_{m}-\delta_{n, m-1} \delta_{m-1}\right),
\end{aligned}
$$

where

$S_{m} \equiv \sigma_{x}^{m} \sigma_{z}^{m+1} \sigma_{y}^{m+2}-(x \leftrightarrow y)$.

Since the AF ground state is invariant under rotations around the $z$-axis

$\left\langle 0\left|S_{m}\right| 0\right\rangle=0$.

Hence, in the $N=\infty$ limit

$\left[J^{1}(x), J^{1}(y)\right]=0$.

We can also compute the chiral anomaly from eq. (17)

$$
\begin{aligned}
& \epsilon^{\mu \nu} \partial_{\mu} J_{\nu}(x) \\
& \quad=\lim _{a \rightarrow 0} \lim _{N \rightarrow \infty}-\frac{4}{8 a^{2}}\langle 0|\left(\sigma_{n}^{x} \sigma_{n+1}^{x}+\sigma_{n}^{v} \sigma_{n+1}^{y}\right) \\
& \quad \times\left(\sigma_{n-1}^{z}-\sigma_{n+1}^{z}\right)|0\rangle .
\end{aligned}
$$

We know that $|0\rangle$ is invariant under the action of the operator $[3,7]$

$\Sigma=\prod_{n=1}^{N} \sigma_{n}^{x}, \quad \Sigma|0\rangle=|0\rangle$

Since the operator in the RHS of eq. (42) anticommutes with $\Sigma$

$\varepsilon^{\mu \nu} \partial_{\mu} J_{\nu}(x)=0$

(when $N=\infty$ ).

Let us now analyse the obtained results. The spacespace [eq. (41)] ETC of the current vanish as in the continuum solutions of the Thirring model [5]. However, our Schwinger term [eq. (36)] depends on the coupling constant $g(g=2 \cos \mu)$ in a non-trivial way. This is not the case in the continuum solutions [5] where one finds $Z \equiv 1$ for all values of the coupling. That is, the value we find only when $g=0$. Let us now see that one cannot explain this fact by assuming that our vector current $J_{\mu}(x)$ and the one in the continuum construction $\left(j_{\mu}(x)\right.$ of ref. [5] ) differ by a coupling dependent normalization.

$J_{\mu}(x) \stackrel{?}{=} \sqrt{Z(\mu)} j_{\mu}(x)$.

The long-distance behavior of the equal-time $J_{0}(n)$ correlations is exactly known [11]

$$
\left\langle 0\left|J^{0}(n) J^{0}(0)\right| 0\right\rangle \underset{x=n a \rightarrow \infty}{=}-\frac{1}{8(\pi-\mu)^{2} x^{2}}
$$

+higher orders.

The continuum construction yields [5]

$\left\langle 0\left|j_{0}(x) j_{0}(0)\right| 0\right\rangle=-1 / 2 \pi^{2} x^{2}$.

We see that both results coincide at $\mu=\frac{1}{2} \pi$. For $\mu \neq \frac{1}{2} \pi$ the mismatch cannot be explained by a finite renormalization $\sqrt{Z(\mu)}$ [eq. (45)] since a look at eq. (37) shows that

$Z(\mu) \neq 1 / 4(1-\mu / \pi)^{2}$ for $\mu \neq \frac{1}{2} \pi$.

A further argument follows from the current-field commutator. We see from eqs. (18) that they have canonical values in our construction as in the continuum one [5].

This excludes a relation like eq. (45).

Let us now discuss the fermionic field at the light of conformal invariant theory.

From ref. [5] one reads its conformal weights

$s=h-\bar{h}=\frac{1}{2}$,

$x=h+\bar{h}=\frac{1}{2}+g_{\mathrm{K}}^{2} / 4 \pi^{2}$,

where $g_{\mathrm{K}}$ is Klaiber's coupling constant for the continuum Thirring model $\left(g \neq g_{\mathrm{K}}\right.$, in general).

The conformal weights of the spin field in the sixvertex model or XXZ chain are known from refs. [13,14]

$x=1 / 8(1-\mu / \pi)+\frac{1}{2}(1-\mu / \pi)$.

Notice that such conformal weights are not realized by physical Bethe Ansatz states [14]. Moreover, we can identify the conformal weights (50) with those given by eq. (49).

Of course the lattice field $\phi_{n}$ has a lot of structure that disappears in the $a=0$ limit. Only its conformal properties are identical to the fermion field in ref. [5]. This leads to the following connection between couplings: 


$$
\begin{aligned}
& \mu / \pi=\frac{1}{2} \\
& \quad+\left(g_{\mathrm{K}} / 2 \pi\right) \sqrt{1+\left(g_{\mathrm{K}} / 2 \pi\right)^{2}}-\left(g_{\mathrm{K}} / 2 \pi\right)^{2}
\end{aligned}
$$

or

$$
\begin{aligned}
g= & +2 \cos \mu \\
& =2 \sin \left(\left(\frac{1}{2} g_{\mathrm{K}}\right) \sqrt{1+\left(g_{\mathrm{K}} / 2 \pi\right)^{2}}+\left(g_{\mathrm{K}} / 2 \pi\right)^{2}\right) .
\end{aligned}
$$

Notice that near the decoupling point

$$
g \underset{g \rightarrow 0}{\simeq} g_{K}+\mathrm{O}\left(g^{2}\right) \text {. }
$$

That is, the field $\Psi(x)$ [eq. (16)] and currents $J_{\mu}(x)$ [eqs. (11), (13)] provide a new operatorial solution with spin $\frac{1}{2}$ of the massless Thirring model not included in previous ones [5]. As conformal model (not as a lagrangian QFT) its content is the same as the one of previous continuous construction.

The results exposed in this paper show that Schwinger terms (ST) do appear in lattice Bethe Ansatz constructions. In the coordinate Bethe Ansatz this usually is not the case. This is connected with the vacuum dependence of such terms. Our derivations [eqs. 25)-(41)] show explicitly that the ST appear only for the physical vacuum and not in the bare one.

We display for the first time in this paper the properties of the Thirring model solution obtained as continuous limit of the XXZ Heisenberg chain. These properties obtained through the Bethe Ansatz show that a new relativistic solution of the Thirring model arises in this way. The current $\left(J_{\mu}\right)$ and field $(\Psi)$ operators cannot be directly connected with the constructions available in the literature.
We want to thank C. Destri for a discussion. One of us (T.J.M.S.) wishes to thank the member of the LPTHE (Univ. Paris VII) for their kind hospitality. TJMS is supported in part by the Conselho Nacional de Desenvolvimiento Cientifico e Tecnologico, Brazil.

\section{References}

[1] L.D. Faddeev, Soc. Sci. Rev. C 107 (1980) 1; Les Houches School Proc. (North-Holland, Amsterdam, 1983); H.B. Thacker, Rev. Mod. Phys. 53 ( 1981 ) 243.

[2] H.J. de Vega, Yang-Baxter algebras, integrable models and quantum groups, LPTHE PAR preprint 88-26; São Paulo Lect. (1987), in: Two dimensional models and string theories, eds. E. Abdalla and M.C.B. Abdalla (World Scientific, Singapore, 1988).

[3] R.J. Baxter, Exactly solved models in statistical mechanics, preprint.

[4] A. Luther, Phys. Rev. B 14 (1976) 2153; M. Lüscher, Nucl. Phys. B 117 (1976) 475.

[5] B. Klaiber, Boulder Lectures in Theoretical physics, Vol. XA (1968) p. 141; J.A. Swieca, Fortschr. Phys. 25 (1977) 303.

[6] H.J. de Vega and E. Lopes, Phys. Lett. B 186 (1987) 180.

[7] H.J. de Vega and F. Woynarovich, Nucl. Phys. B 251 (1987) 439.

[8] M. Gaudin, B.M. McCoy and T.T. Wu, Phys. Rev. D 23 (1981) 417

[9] K. Odaka and S. Tokitake, Tsukuba UTHEP preprint (1987).

[10] C.N. Yang and C.P. Yang, Phys. Rev. 150 (1966) 321, 327.

[11] A. Luther and I. Peschel, Phys. Rev. B 12 (1975) 3918; N.M. Bogolubov, A.G. Izergin and V.E. Korepin, Nucl. Phys. B 275 (1986) 687 .

[12] L.P. Kadnoff and A.C. Brown, Ann. Phys. (NY) 121 (1979) 318.

[13] H.J. de Vega and M. Karowski, Nucl. Phys. B 285 (1987) 619

M. Karowski, Nucl. Phys, B 300 (1988) 473. 